Ann. Génét. Sél. anim., 1970, 2 (3), 263-267.

\title{
GÈNES A EFFET VISIBLE ET COLORATION OU ÉPAISSEUR DES COQUILLES D'GEUFS
}

\author{
P. MÉRAT
}

avec la collaboration technique de A. Bordas, G. Coquerelle et L. Durano

Station centrale de Génétique animale, Centre national de Recherches zootechniques, 78-Jouy-en-Josas

Institut national de la Recherche agronomique

\section{RÉSUMÉ}

Parmi 12 loci étudiés dans une population, deux, concernant la coloration du plumage, présentent une association avec l'épaisseur et la pigmentation externe des coquilles d'œufs: les poules à plumage entièrement noir comparées à celles à noir restreint (locus $E$ ) et les poules colorées (ii) comparées à celles où le noir est absent du plumage (Ii) pondent des oufs à coquille significativement plus épaisse et de teinte plus foncée. Une tendance analogue mais moins marquée semble exister, quant à l'épaisseur des coquilles, pour deux autres loci $(C$ et $S)$ de pigmentation du plumage.

\section{INTRODUCTION}

Nous avons fait état à diverses reprises d'associations trouvées dans notre cheptel entre des gènes identifiés par un effet extérieur et des performances quantitatives, et résumé à ces occasions les résultats obtenus par d'autres chercheurs (MÉRAT, I962, I967).

A notre connaissance, l'on n'a pas recherché de telles associations avec des caractéristiques des œufs autres que le poids. Tel est l'objet du présent travail.

\section{MATÉRIEL ET MÉTHODES}

Des détails sur la population étudiée, contenant divers gènes maintenus en ségrégation, sont contenus dans nos articles antérieurs (MÉRAT, 1962). 
Pour ces gènes, la comparaison, relative à un caractère quantitatif, des moyennes des génotypes hétérozygote et homozygote récessif est faite sur des couples de sœurs écloses le même jour et élevées ensemble.

Les loci pour lesquels des nombres de couples suffisants ont été obtenus en groupant plusieurs années (1964 à 1968), sont les suivants; $C, I, E, S, B l, W, K, P o, N a, R, P, H e$ (CAvalie et Mérat, 1963). A part He, les symboles utilisés sont ceux de Hutr (1949).

Les poules étant placées en poulaillers de ponte au sol, les caraetères suivants sont relevés sur des œufs consécutifs pondus à 10 mois d'âge avant la mise en reproduction :

- épaisseur de la coquille ( $y$ compris la membrane coquillière), mesurée au $1 / 100$ millimètre vers l'équateur de l'œuf par un comparateur, chaque poule étant caractérisée par la moyenne de deux cufs consécutifs;

- hauteur de l'albumen épais, au 1/10 de millimètre sur l'œuf cassé et étalé sur une surface vitrée plane (mesure faite sur 2 œufs frais par poule, avec un micromètre à trépied);

- coloration brune de la coquille, notée de 1 à 9 par comparaison avec une échelle témoin renouvelée chaque année (sur 5 cufs par poule).

Les hauteurs d'albumen sont utilisées sans correction du type "unités Haugh ", les différences moyennes de poids d'œufs entre génotypes étant trop faibles pour influer sur le résultat des comparaisons.

Les nombres d'œufs utilisés par poule pour chaque mesure seraient faibles pour une évaluation précise de sa valeur individuelle, mais ils suffisent au but recherché, comparaison de génotypes différents.

\section{RÉSULTATS ET DISCUSSION}

Les résultats les plus significatifs concernent l'épaisseur des coquilles. Deux loci, $\mathrm{E}$ et $\mathrm{I}$, révèlent des écarts nets entre génotypes (tableau I).

TABLEAU I

Différences d'épaisseur des coquilles associées à des gènes à effet visible

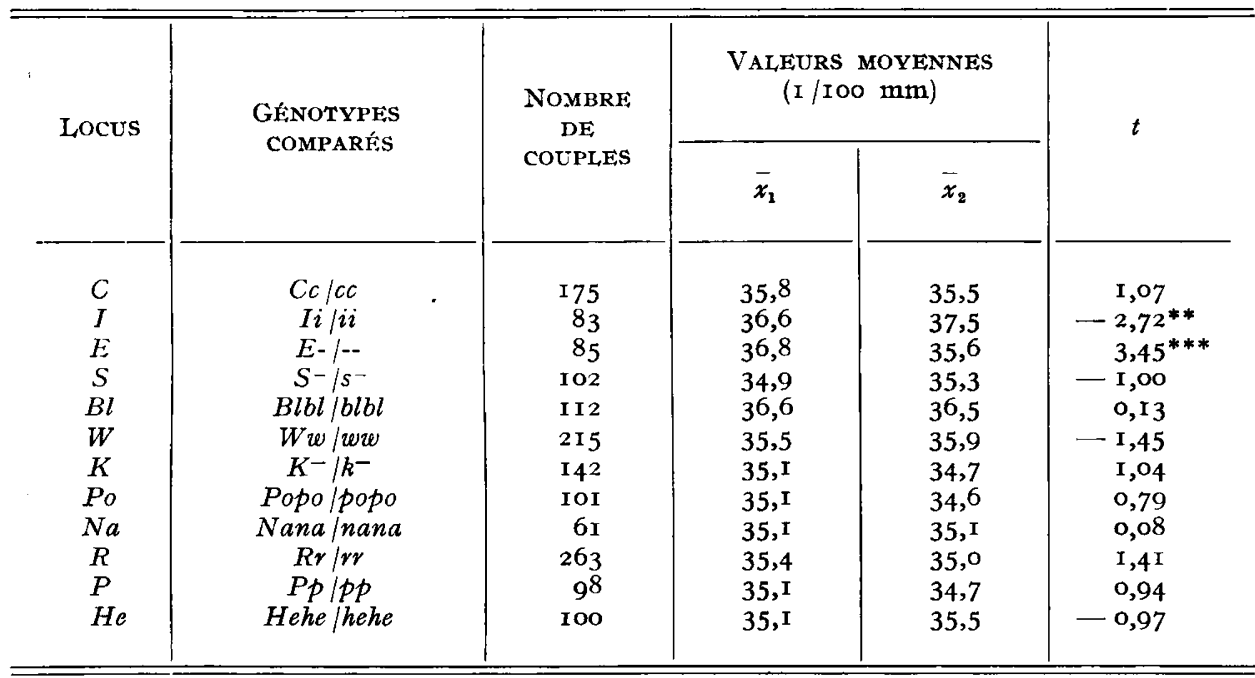

Le locus $E$ comportant des allèles multiples (Sмyтн, r969), la comparaison $\mathrm{y}$ est faite entre animaux possédant un gène $E$ (" noir étendu ") ou non (" noir restreint ").

Pour les deux loci, les poules au plumage plus pigmenté produisent vers Io mois d'âge des coquilles légèrement mais très significativement plus épaisses. 
On peut noter qu'avec deux autres loci de coloration du plumage étudiés dans cette population, le génotype le plus "mélanisé " est également associé à la présence de coquilles plus épaisses en moyenne, quoique les différences ne soient pas significatives : 35,8 pour les poules colorées $C c$ contre 35,5 pour les blanches $c c$ sur I 75 couples, et 35,3 pour les $s$ - ("dorées ") contre 34,9 pour les $S$ - ("argentées ») sur I02 couples. Par contre, la différence est négligeable au locus $B l$ de dilution de la coloration du plumage.

Pour l'intensité de la couleur de coquille (tableau 2), les poules à plumage à noir étendu, hétérozygotes pour $E$, ont une note moyenne supérieure à celle de leurs sœurs à noir restreint : 4,5 pour les premières contre 4,0 pour les secondes, sur 85 couples $(\mathrm{P} \simeq 0,05) \quad\left({ }^{1}\right)$. La différence correspondante n'est significative à aucun autre locus, mais la coloration moyenne est un peu plus intense chez les $C c$ que chez les $c c(5,4$ contre $5, \mathrm{I})$ de même que pour les $i i$ par rapport aux $I i$ $(4,2$ contre 3,9$)$, la différence étant très faible aux autres loci de coloration du plumage, $S$ et $B l$.

TABLEAU 2

Différences de pigmentation des coquilles associées à des gènes à effet visible

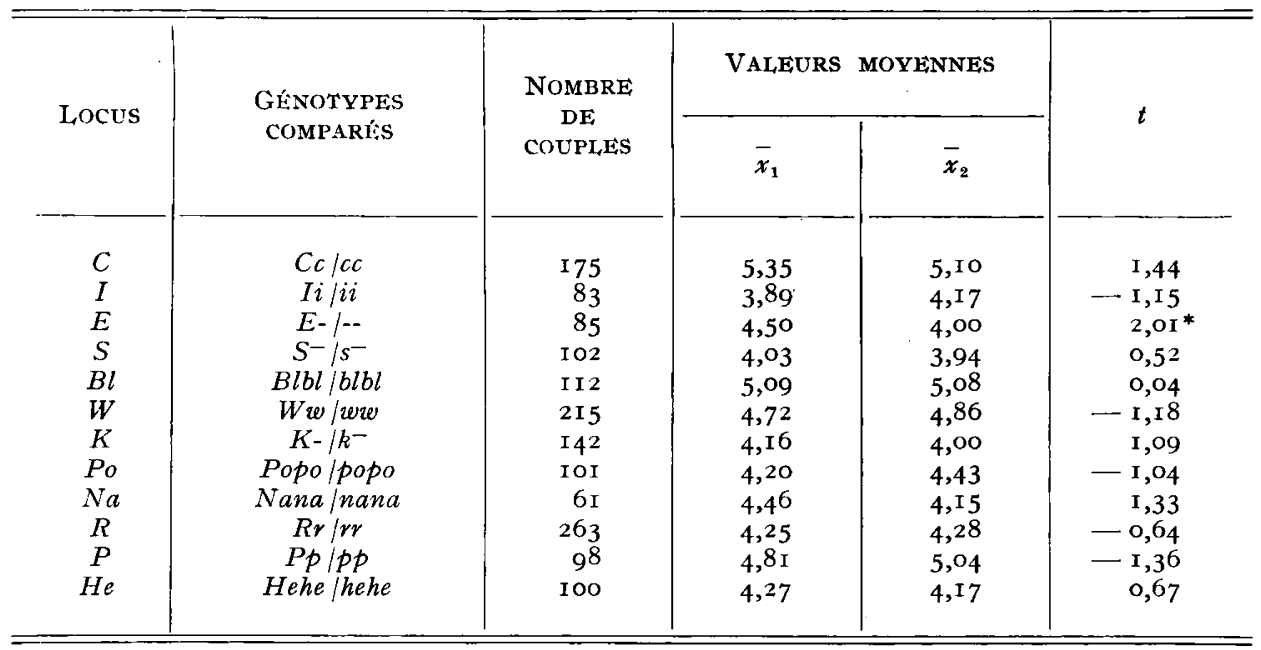

Dans 1'ensemble, les résultats relatifs à la teinte des coquilles semblent donc assez parallèles à ceux se rapportant à leur épaisseur. Quant à la hauteur de 1'albumen (tableau 3), la seule différence significative concerne le locus $P o$ (hauteur de l'albumen inférieure pour les poules à 5 doigts).

Les variances, quant à elles, ne présentent pour aucun locus de différence caractérisée suivant le génotype.

Dans certains cas, (loci $C, I, R, P$ ), les données comportaient les croisements o $A a \times$ i $a a$ et $\delta a a \times+A a$, de sorte qu'une recherche d'effets maternels éven-

(1) Le test $t$ peut s'appliquer, les distributions des notes individuelles, moyennes de 5 œufs, étant approximativement normales. 
TABLEAU 3

Différences de hauteur de l'albumen associées à des gènes à effet visible

\begin{tabular}{|c|c|c|c|c|c|}
\hline \multirow{2}{*}{ Locus } & \multirow{2}{*}{$\begin{array}{l}\text { GÉNOTYPES } \\
\text { COMPARÉS }\end{array}$} & \multirow{2}{*}{$\begin{array}{c}\text { NOMBRE } \\
\text { DE } \\
\text { COUPLES }\end{array}$} & \multicolumn{2}{|c|}{$\begin{array}{l}\text { VALEURS MOYENNES } \\
\text { (I / IO } \mathrm{mm})\end{array}$} & \multirow{2}{*}{$t$} \\
\hline & & & $\bar{x}_{1}$ & $\bar{x}_{2}$ & \\
\hline C & Cc/cc & & 667 & & 89 \\
\hline$I$ & Ii $/$ ii & 83 & 6,74 & 6,47 & I, 68 \\
\hline$E$ & $E-1--$ & 85 & 6,60 & 6,50 & 0,65 \\
\hline$S$ & $S-/ s$ & 102 & 6,08 & 6,12 & $-0,26$ \\
\hline$B l$ & $B l b l / b l b l$ & 112 & 6,01 & 6,16 & $-0,87$ \\
\hline$W$ & $W w / w w$ & 215 & 6,67 & 6,55 & I,45 \\
\hline$K$ & $K-/ k-$ & 142 & 5,96 & 6,03 & $-0,63$ \\
\hline Po & Popo/popo & IOI & 6,13 & 6,54 & $-2,54^{* *}$ \\
\hline $\mathrm{Na}$ & Nana/nana & $6 r$ & 6,37 & 6,44 & $-0,76$ \\
\hline$R$ & $R r / r r$ & 263 & 6,23 & 6,27 & $-0,56$ \\
\hline$P$ & $P p \mid p p$ & 98 & 6,84 & 6,60 & $x, 48$ \\
\hline $\mathrm{He}$ & Hehe /hehe & 100 & 6,17 & $6,1 \mathrm{I}$ & 0,49 \\
\hline
\end{tabular}

tuels était possible par comparaison entre ces croisements réciproques (analyse de variance sur les moyennes des pères, avec pour facteurs contrôlés le type de croisement et l'année). Au locus $P$, un tel effet maternel est suggéré, les filles de mères $P P$ pondant des œufs à blanc plus épais $(P<0,05)$ et à coquille plus teintée $(P \simeq 0,02)$. Cependant, le nombre total de familles en jeu n'est pas encore très élevé et cette conclusion doit être tenue pour provisoire.

$\mathrm{Au}$ total, pour I2 loci étudiés et 3 caractères, 4 comparaisons entre génotypes individuels sont significatives au-delà du seuil 5 p. Ioo. On pourrait s'interroger a priori sur la réalité des différences correspondantes. Cependant, l'effet sur l'épaisseur de la coquille associé au locus $E$, significatif au-delà du seuil I p. I ooo, aurait une faible probabilité d'être observé par hasard même parmi l'ensemble de toutes les comparaisons. D'autre part, le parallélisme des résultats sur plusieurs gènes responsables de la présence ou de l'extension des mélanines dans le plumage, quant à l'épaisseur des coquilles, semble suggérer une relation physiologique entre cette dernière et le degré de " mélanisation " de l'animal, quoique nous n'ayons pas d'explication sur la nature de cette relation. Il faut rappeler que notre mesure d'épaisseur inclut la membrane coquillière; des mesures séparées de cette dernière seront utiles pour une interprétation plus précise de nos résultats.

Quant aux résultats qui semblent indiquer aussi une association positive entre coloration du plumage et intensité de la pigmentation brune de l'œuf, attribuable à des porphyrines (HưT, I949), il est possible qu'ils reflètent la corrélation trouvée à plusieurs reprises (HUTT, I949, MANNELS et SAUNDERS, I965) entre épaisseur et coloration de la coquille.

Reçu pour publication en mai I97o. 


\section{REMERCIEMENTS}

Nous remercions MM. Legault, Station de Génétique animale, C.N.R.Z., Jouy-en-Josas, et Lacassagne, Station de recherches avicoles, C.R.V.Z., Nouzilly, pour leurs remarques et critiques concernant la présentation de cet article.

\section{SUMMARY}

\section{GENES WITH VISIBLE EFFECT AND THICKNESS \\ OR EXTERNAL, PIGMENTATION OF EGGSHELLS}

Among 12 loci studied in one population, two, concerned with plumage color, show an association with thickness and external pigmentation of eggshells : Pullets with extended black compared with restricted black plumage ( $E$ locus) and pullets with colored (ii) vs non-black plumage $(I i)$ lay eggs with significantly thicker and more intensely pigmented shells. A similar but less marked tendency seems to be associated, for shell thickness, with two other plumage color loci $(C$ and $S$ ).

\section{RÉFÉRENCES BIBLIOGRAPHIQUES}

HutT F. B., I949. Genetics of the fowl. Mc Graw Hill, New-York.

Mannels K., Saunders C., r965. (Poids spécifique et couleur de la coquille des oufs de poules australorp noires). S. Afr. J. agric. Sci., 8, 961-968.

MÉrat P., 1962. Quelques relations entre caractères extérieurs à hérédité simple et productivité. Proc. $X I I t h$ World's Poultry Congress, Sydney, Section papers, $7 \mathrm{r}-76$.

Mérat P., I967. Contribution à l'étude de la "valeur sélective " associée à quelques gènes chez la Poule domestique. Annls biol. anim. Biochim. Biophys., 7, 79-104.

Suyтн J. R., Jr., 1969. Relationship between genes affecting melanin pigmentation and other traits in the fowl. World's Poult. Sci. J., 25, 6-14. 